\title{
Control of vascular network location in millimeter-sized 3D-tissues by micrometer-sized collagen coated cells
}

\author{
Chun-Yen Liu, Michiya Matsusaki and Mitsuru Akashi
}

Dr. Chun-Yen Liu, Dr. Michiya Matsusaki

Department of Applied Chemistry, Graduate School of Engineering, Osaka University, 2-1 Yamadaoka, Suita, Osaka 565-0871, Japan.

Prof. Mitsuru Akashi

Graduate School of Frontier of Biosciences, Osaka University

1-3 Yamadaoka, Suita, Osaka 565-0871, Japan

E-mail: akashi@fbs.osaka-u.ac.jp

Keywords: tissue engineering, coating, collagen, endothelial cells, vascularization,

location control

\begin{abstract}
Engineering three-dimensional (3D) vascularized constructs remains a central challenge because capillary network structures are important for sufficient oxygen and nutrient exchange to sustain the viability of engineered constructs. However,
\end{abstract} construction of 3D-tissues at single cell level has yet to be reported. Previously, we established a collagen coating method for fabricating a micrometer-sized collagen matrix on cell surfaces to control cell distance or cell densities inside tissues. In this study, a simple fabrication method is presented for constructing vascular networks in 3D-tissues over micrometer-sized or even millimeter-sized with controlled cell densities. From the results, well vascularized 3D network structures can be observed with a fluorescence label method mixing collagen coated cells and endothelia cells, 
indicating that constructed ECM rich tissues have the potential for vascularization, which opens up the possibility for various applications in pharmaceutical or tissue engineering fields.

\section{Introduction}

Living tissues and organs form well-organized 3D structures with different types of cells and ECMs. These usually have complex mass transportation systems which are important for acquiring oxygen, nutrients, or even removing the metabolic by-products from the tissues or organs. Recently, construction of bio-mimetic living tissues or organ models with various highly organized cells and extracellular matrices (ECMs) have attracted increasing attention in the fields of tissue engineering and pharmaceutical assessment [1]. In particular, the fabrication of vascularized, thick and complex tissues is still a key challenge for transportable constructs. To construct such vascularized 3D-tissues, several bottom-up approaches like cell sheet [2-4], hydrogels composed of extracellular matrix (ECM) [5-7], micro channel models with flow [811], and angiogenic factors fixed scaffolds [12,13], have been reported for the construction of 3D-tissues. Nevertheless, these techniques have their limitations because of the complicated devices or procedures needed for construction, and the recreation of functions and structures of living tissues in vitro still elusive. 
Recently, our research group developed a simple and unique bottom-up approach called "hierarchical cell accumulation" by preparing nanometer-sized films on cell surfaces using the "Layer-by-Layer" (LbL) concept developed by G. Decher in 1981 $[14,15]$. Approximately $6 \mathrm{~nm}$-thick FN-G films were fabricated on cell surfaces by coating fibronectin (FN) and gelatin (G) alternatively for 9 steps [16-18]. A variety of multi-layered 3D-tissues using different kinds of cells can be fabricated. Furthermore, we also reported on the "cell accumulation technique", to fabricate tissues over 100 $\mu \mathrm{m}$ thick with blood capillary networks $[17,18]$. However, the construction of centimeter-sized level 3D-tissues with controlled 3D-cell density, 3D-cell component, and 3D-cell location remains a challenging problem. Previously, we reported a novel method to fabricate a micrometer-sized level collagen scaffold onto cell surfaces by utilizing fiber formation of type I collagen [21-23], which is the most abundant ECM in the human body [24]. Collagen Type I collagen can be recognized by $\alpha 2 \beta 1$ Integrin receptors located on cell membranes [25], giving strong interactions (association constant of $6.7 \times 10^{4} \mathrm{M}^{-1}$ ) [26] and firm interconnections on cell membranes [27]. Until now, about $100-2,000 \mu \mathrm{m}$ thick 3D-tissues with lower cell densities or varied cell densities were constructed successfully by utilizing various sizes of collagen coated cells and seeding cell numbers [28]. Besides, Functionalized 3D tissues can also be constructed by induced pluripotent stem derived cardiomyocytes. As we know, 
tissue thicknesses greater than $200 \mu \mathrm{m}$ require a capillary network system for sufficient nutrient, oxygen, and even waste exchange inside the thick tissues to support normal physiological functions of the cells [29,30]. Accordingly, in this study, we describe a simple method for constructing micrometer-sized to millimeter-sized 3D-tissues with blood capillary network structures by using collagen coated cells in engineered tissues (Figure 1). We hypothesized that the tissues constructed by collagen coated cells can promote the formation of blood vessels inside the tissues and provide functional support for the tissues.
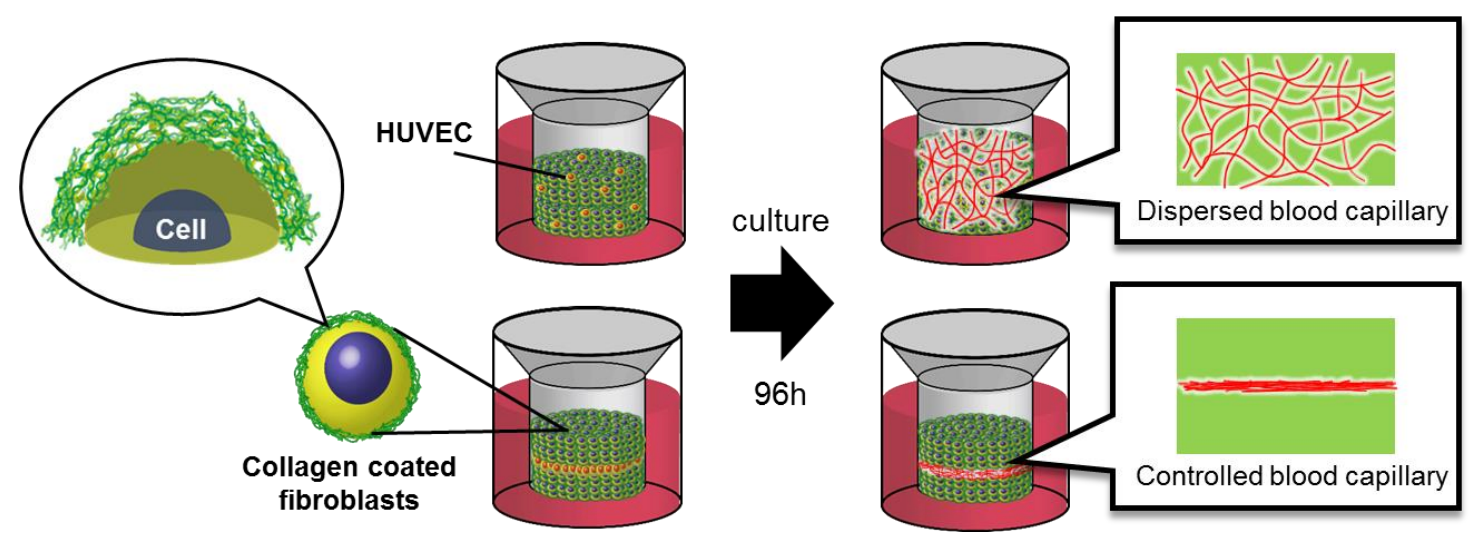

Figure 1. Schematic illustration of coating collagen nanofiber matrices onto cell surfaces and application visions.

\section{Experimental Section}

\subsection{Materials}

Human dermal fibroblast cells were purchased from CAMBREX (East Rutherford, USA). Human Umbilical Vein Endothelial Cell (HUVEC) was purchased from Lonza 
(NJ, USA). Type I collagen solution (from bovine corium, $0.3 \mathrm{wt} \%, 5 \mathrm{mM}$ acetic acid) was purchased from Nippi Co. Ltd. Dulbecco's modified eagle medium (DMEM), $10 \%$ formalin solution (4\% formaldehyde in water containing methanol), $4 \%$ paraformaldehyde (PFA), trypsin, and $1 \mathrm{M} \mathrm{NaOH}$ were purchased from Wako Pure Chemical Industries, Ltd (Osaka ,Japan). 5x DMEM was purchased from Biological Industrials (Kibbutz Beit Haemek, Israel). Fetal Bovine Serum (FBS) was purchased from Biowest (Miami, USA). The antibiotics were purchased from Nacalai Tesque (Kyoto, Japan). The monoclonal mouse anti-human CD31 antibodies were purchased from Dako (Glostrup, Denmark). Goat anti-mouse Alexa Fluor 546-conjugated IgG, 40,6-diamidino-2-phenylindole dihydrochloride (DAPI) and Triton-X, were purchased from Life Technologies (CA, USA). The culture medium, Petri dish and centrifugation tube were purchased from AGC Techno Glass Co. The plastic tube was purchased from Sumitomo Bakelite (Tokyo, Japan). All reagents were used without further purification.

\subsection{Cell culture}

Normal human dermal fibroblast (NHDF) cells at passages 5-9 were cultured in DMEM with 10\% FBS and 1\% antibiotics; HUVEC at passages 5-8 were cultured in Endothelial Cell Growth Medium (EGM-2) at $37{ }^{\circ} \mathrm{C}$ in a humidified $5 \% \mathrm{CO}_{2}$ 
incubator. During each passage, cells were treated with $3 \mathrm{~mL}$ of $0.1 \%$ trypsin for 3 minutes to detach them from the culture media. Detached cells were then collected in a $15 \mathrm{~mL}$ plastic tube containing $4 \mathrm{~mL}$ of DMEM and centrifuged at 1,000 rpm for 5 min. The supernatants of the centrifuged solutions were removed, and fresh DMEM was added into the plastic tube for calculating the cell numbers or for use in further experiments.

\subsection{Fabrication of collagen nanofiber matrices on cell surfaces}

In this study, NHDFs coated with micrometer-sized collagen nanofiber matrices were applied to construct 3D-tissue models with lower cell densities. Before coating collagen nanofiber matrices on cell surfaces, NHDFs at passages 5-9 underwent trypsinization for 20 minutes to produce spherical cells. Cells retained high cell viability after the trypsinization procedures. Detached cells were then mixed with 0.03 wt $\%$ collagen coating solutions containing FBS, 5x DMEM at $37{ }^{\circ} \mathrm{C}$ with rotation $(50$ rpm, TAITEC, Japan) for 20 minutes. The coating solutions were washed twice with Phosphate Buffered Saline (PBS) to remove any impurities, and then collected by centrifugation at 1,000 rpm for 5 minutes. The resulting collagen coated cells were then suspended in fresh DMEM and the same coating procedures were repeated to fabricate about $15 \mu \mathrm{m}$-thick collagen nanofiber matrices on cell surfaces. Finally, all 
collagen coated cells with $3 \mu \mathrm{m}$ and $15 \mu \mathrm{m}$-thick collagen layers were used to further the tissue construction process.

\subsection{Transmission Electron Microscope (TEM) observation}

To understand the relationship between collagen nanofibers and cells in detail, cells without coating and fabricated collagen coated cells with different sizes were observed by Transmission Electron Microscope (TEM) in this study. Firstly, cells with and without coating with collagen nanofiber matrices ( 1 coat and 2 coats) were fixed in $2.5 \%$ glutaraldehyde $/ 10 \%$ formalin buffer and post-fixed in $2 \%$ osmium tetroxide solution, dehydrated in a graded ethanol series, and then embedded in Epon 812 (Oken, Tokyo, Japan). Ultrathin sections were prepared, stained with uranyl acetate and lead citrate, and observed under a JEM-1200 EX TEM (JEOL, Tokyo, Japan).

\subsection{Protection of collagen layers on cell surfaces under dried conditions}

As we know, the injection printing method is one of the usual methods for constructing 3D-tissue models. Cell location can be precisely controlled at a desired location after propulsion from the nozzle. In this situation, water loss from the cells always occurs during the construction process, which may lead to cell death because of a lack of moisture. To prevent cell death caused by water loss, we theorized that 
collagen coated cells might decrease the speed of water loss from the cells. To this end, cells with (1, 2, and 3 coats) and without coating were applied to evaluate cell viability at room temperature and inside the incubator for various lengths of time. Briefly, cells with and without coating were placed on a cover glass for a certain length of time and the cell viability was then evaluated using a trypan blue assay.

\subsection{Construction of the blood capillary model using a mixing method}

As shown in Figure S1(a), a mixing method was applied to construct 3D-tissue models with vascularized structures in this study. Similar to the NHDFs detachment procedures, HUVECs underwent trypsinization at $37{ }^{\circ} \mathrm{C}$ for about 20 minutes, following resuspension in fresh DMEM. About 10\% and 20\% of HUVECs (compared with total cell numbers) and collagen coated NHDFs prepared in the previous section were mixed together and then seeded in 24-well culture inserts to construct 3D-tissues ( 2 and $4 \times 10^{6}$ cells in total). Culture inserts were pre-coated with $0.2 \mathrm{mg} / \mathrm{ml}$ human plasma fibronectin (FN) for 30 minutes to enhance interactions between insert membranes and collagen coated cells before seeding the cells [15]. Then fresh DMEM was added into the well and the mixture was incubated in an incubator after seeding. Another fresh DMEM was added after an hour to cover the culture inserts in order to supply sufficient nutrients to the 3D-tissues. All constructed tissues were cultured for 
3 days without changing media and then fixed with 4\% PFA buffer solutions for further analyses.

\subsection{Construction of the blood capillary model using a sandwich culture method}

Until now, there have been no reports about construction of capillary network structures in millimeter-sized tissues under lower cell densities. Hence, we would like to construct 3D-tissues using a sandwich culture method (Figure S1(b)). Similar to the previous published method, about $1 \times 10^{6}$ cells and $2 \times 10^{6}$ cells ( 1 or 2 coats) were used to seed in $0.2 \mathrm{mg} / \mathrm{ml}$ human plasma fibronectin (FN) coated insert for 24 hours as the first layers. About $6 \mathrm{ml}$ of DMEM was added into the 6-well plate for an hour, and another $6 \mathrm{ml}$ of DMEM was added to cover the insert for sufficient supply of nutrients. 10\% HUVECs (compared with total cell numbers) were used to seed onto the first constructed NHDF layers as the second layers on the following day. Then, additional collagen coated cells ( 1 coat or 2 coats) with same seeding cell numbers as the first layer were seeded on the second layers as the third layers. A total of $12 \mathrm{ml}$ of DMEM was added to the well during construction of the second and third layers. The constructed tissues were incubated for 3 days without changing culture media. Tissues were then fixed with 4\% PFA buffer solutions for further analyses. 


\subsection{Oxygen partial pressure measurement}

The oxygen partial pressure in the media were measured with an oxygen partial pressure meter (PO2-150S, Eikoukagaku, Tokyo, Japan). The current value of oxygen on the surface of the electrode (POE-20W, Eikoukagaku, Tokyo, Japan) under a suitable voltage using the polarography principle for estimating oxygen partial pressure was measured by this device. Oxygen partial pressure of all constructed tissues were measured continuously for 36 hours after seeding. The culture media were changed after 24 hours incubation. The starting value of oxygen partial pressure without tissue was set at $150 \mathrm{mmHg}$.

\subsection{Immunofluorescent analysis}

In this experiment, endothelial cells (HUVECs) were immunostained with an anti-CD31 antibody, and all cell nuclei were immunostained with DAPI. Briefly, the fixed tissues were permeabilized with $0.2 \%$ Triton-X for 30 minutes and blocked with $1 \%$ BSA/PBS for one hour. The tissues were then incubated with the primary antibody anti-CD31 mouse (1:100) for a further hour at room temperature. After washing three times with PBS, secondary antibody goat anti-mouse Alexa Flour 546 (1:100) was added to the tissues for one hour at room temperature. In the final step, DAPI (1:1000) was applied to the tissues overnight at $4{ }^{\circ} \mathrm{C}$ after washing three times 
with PBS. The stained tissues were observed by CLSM. The CLSM images were collected and modified by Fluoviewer 4.0.

\subsection{Histological analyses of constructed 3D-tissues}

To ascertain the integrity of constructed tissues, histological analyses were applied in this study. Constructed 3D-tissues were rinsed with PBS solution, and then immersed in $10 \%$ formalin solution at room temperature for 24 hours. Fixed samples were then cut out and embedded in paraffin. The embedded samples were sliced and underwent hematoxylin-eosin (HE) staining. The sectioned tissues were observed using phase contrast microscopy (CLSM FLUOVIEW FV10i and EVOS® XL digital microscope), and the thickness of the constructed 3D-tissues was measured using desktop ruler software and calculated via Microsoft Excel.

\section{Results \& Discussion}

\subsection{Transmission Electron Microscope (TEM) observation}

In previous reports, we observed the collagen nanofiber matrices coated cells using a Scanning Electron Microscope. We observed a multitude of collagen nanofibers spread out on the cell surfaces. In this study, we used TEM to observe the 
structural detail between cells and collagen. The architecture of cells without coating, cells with 1 coat and 2 coats of collagen nanofiber matrices were all observed.

As shown in Figure S2(a), cells without coating revealed dispersed conditions after fixation. Under 10,000x magnification, cytoskeleton and cytoplasmic protrusion inside the cells could be observed clearly. Some aggregation of collagen nanofiber coated cells could be observed after the first coating, as shown in Figure S2(b), collagen filled the space between two adjacent collagen coated cells. Furthermore, some collagen nanofibers could be seen on the cell surfaces. As noted with the 1-coat cells, cells coated twice with collagen nanofiber matrices also revealed some aggregation results after coating (Figure S2(c)). Some collagen fibrils and filaments were observed. From TEM results, collagen fibers attached to the cell surfaces could be observed clearly. Collagen nanofiber matrices adsorbed onto cell surfaces well, connecting the two adjacent cells together, suggesting that a lot of collagen had accumulated inside the space between the cells.

\subsection{Protection of collagen layers on cell surfaces under dried conditions}

To confirm whether or not collagen matrices on cell surfaces can reduce water loss from cells, cells without coating and cells coated with collagen molecules $(1,2$, and 3 coats) were applied. In this part, cells were placed on cover glasses for various 
lengths of time in a generic environment or inside an incubator. The cell viability was measured by trypan blue assay during experiments. The results shown in Figure S3 (a) indicate cell viability of cells without coating reduced dramatically from $100 \%$ to $0 \%$ within $15 \mathrm{~min}$ in the generic environment. In contrast, cells coated with different thicknesses of collagen matrices showed a greater induration ability under the same conditions. There were concomitant increases in the observed induration time as the thickness of the collagen matrices on cell surfaces increased. Although different thicknesses of collagen matrices showed different abilities to endure negative conditions, all cells died after 20 min deposition in the generic environment. This may be due to the collagen matrices becoming excessively dry during the $15-20 \mathrm{~min}$, resulting in $100 \%$ cell death at around 20 min deposition.

If the samples were placed inside the incubator with high humidity, we found that the cell death speed was greatly reduced. Figure S3 (b) shows that cells without coating retained high cell viability even after $30 \mathrm{~min}$. Once the cells were coated with collagen matrices ( 3 coats), the induration time was prolonged by about 3 times compared to outside the incubator. These results suggest that collagen matrices on cell surfaces provide protection ability to slow the water loss from cells, providing more time for the construction of 3D tissue models under normal conditions. 


\subsection{Construction of the 3D-tissues with blood capillary network structures}

In this study, we applied two different methodologies to construct 3D-tissues with vascular network structures in this report. As shown in Figure S1, a mixing method and a sandwich culture method were applied to construct 3D-tissues with vascular network structures. After 96 hours of incubation, all 3D constructs from these two different construction methods were fixed with $4 \%$ PFA and then immunostained by CD31 to observe the vascular network structures inside the 3D tissues.

At first, we examined the distribution of vascular network structures inside the tissues using a histological slicing method. After fixation and immunostaining steps, two types of samples both underwent histological slicing procedures. The samples were embedded in paraffin and then sliced into $20 \mu \mathrm{m}$ thick samples using a histological slicer. The results of mixing samples constructed by collagen coated cells ( 2 coats) with $2 \times 10^{6}$ cells and $4 \times 10^{6}$ cells are shown in Figure 2(a) and Figure 2(b), seeding HUVECs in upper and lower samples were about $10 \%$ and $20 \%$ compared to total seeding cell numbers. The brown color shown in the photos represents vascular structures. In both 20-layer and 40-layer samples, more brown color can be observed in the $20 \%$ HUVECs samples, whereas less brown color is observable in the $10 \%$ HUVECs samples. Furthermore, the thickness of constructed 3D-tissue models remained stable at about $200 \mu \mathrm{m}$ and $700 \mu \mathrm{m}$ in the 20-layer and 40-layer samples 
respectively, with different seeding ratios of HUVECs.
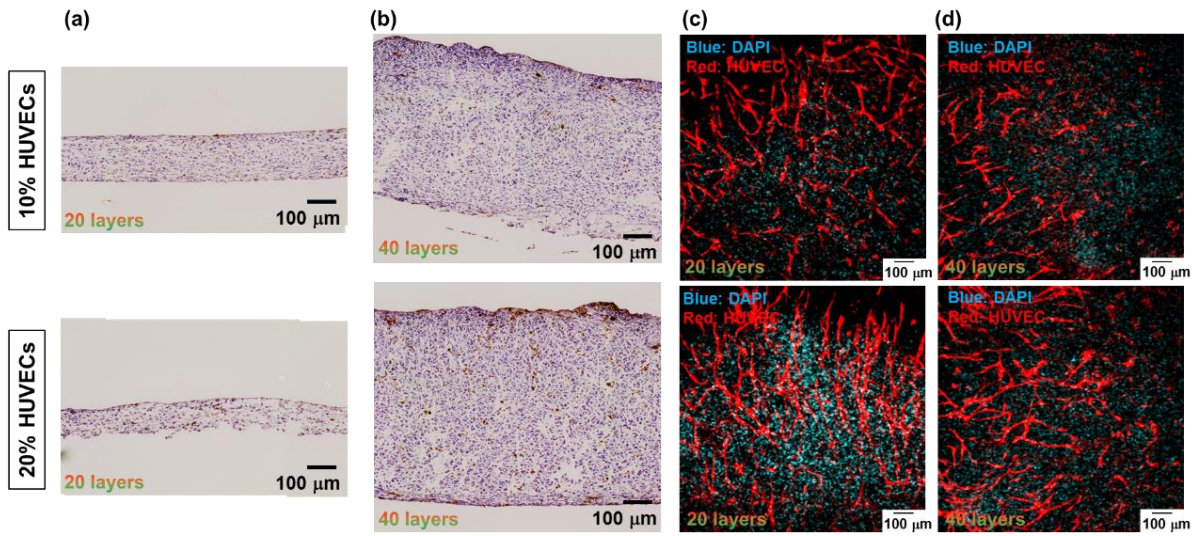

Figure 2. Histological images of 3D-tissues with CD31 immunostaining constructed by (a) 20 layers and (b) 40 layers of collagen coated cells (2 coats) mixed with (upper row) $10 \%$ and (lower row) 20\% HUVECs. CLSM images of mixed 3D-tissues with CD31 immunostaining constructed by (c) 20 layers and (d) 40 layers collagen coated cells ( 2 coats) with $10 \%$ or $20 \%$ HUVECs.

In the case of fluorescence observation, samples underwent fixation and immunostaining with antibodies and fluorescent (Alexa 546), following CLSM observation. As shown in Figure 2(c) and 2(d), vascular network structures (red color) inside the $3 \mathrm{D}$ tissue models constructed by collagen coated NHDFs $\left(2\right.$ coats, $2 \times 10^{6}$ and $4 \times 10^{6}$ cells) with $10 \%$ or $20 \%$ HUVECs were observed. Furthermore, 3D tissues in the $20 \%$ HUVECs samples showed more complex networks than those in the $10 \%$ HUVECs samples under the same seeding cell numbers in one 3D construct, suggesting that network structures can also be controlled. The results suggest that more HUVECs inside near millimeter-sized 3D constructs resulted in more complex networks formed during 96 hours of incubation, which also suggests that the amount of networks may also be controlled inside the thick tissue models. 


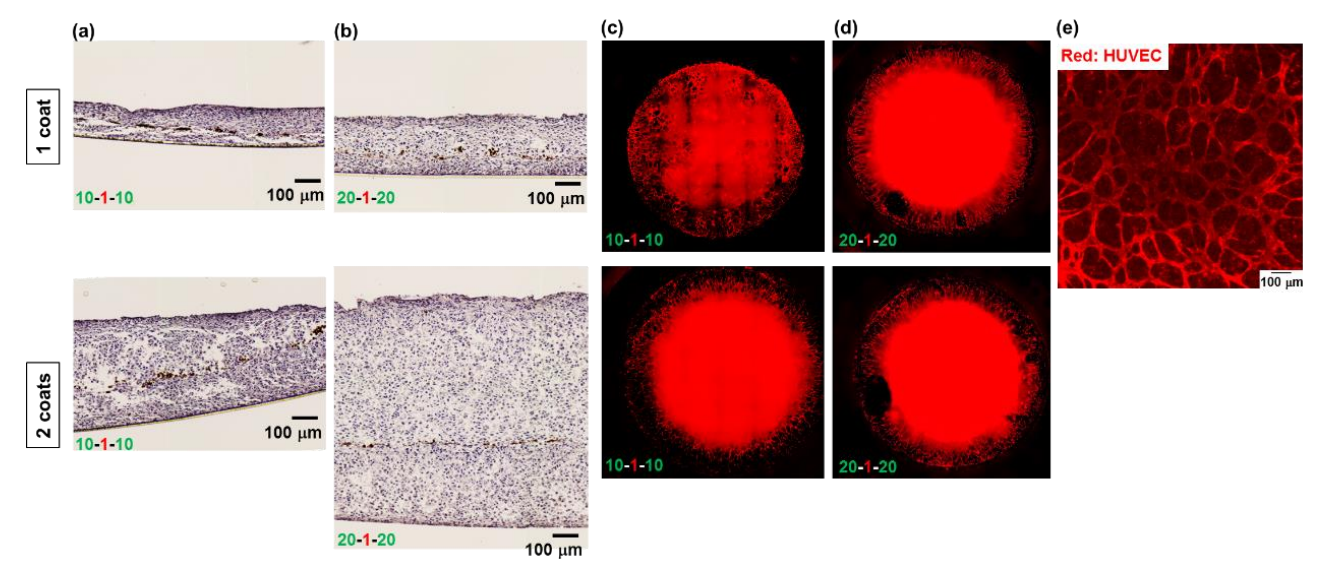

Figure 3. Histological images of 3D-tissues with (a) 10-1-10 and (b) 20-1-20 structures constructed by collagen coated NHDFs ( 1 coat and 2 coats) using sandwich culture method were also obtained. (c)(d) CD31 immunostained images of constructed samples observed by fluorescence microscope. (e) shows well spread blood capillary network structures inside the constructed 10-1-10 tissues (1 coat).

3D-tissue models constructed using a sandwich culture method were also examined by histological and immunostaining methods. Tissues constructed by collagen coated cells ( 1 and 2 coats) and HUVECs following 10-1-10 and 20-1-20 order were also constructed. As shown in the upper photos in Figure 3(a) and Figure 3(b), about $170 \mu \mathrm{m}$ and $200 \mu \mathrm{m}$ thick 3D-tissues with vascular network structures between two collagen coated NHDFs layers ( 1 or 2 coats, 1 × $10^{6}$ cells) were constructed. Once the NHDFs layers increased from $1 \times 10^{6}$ cells to $2 \times 10^{6}$ cells, about $200 \mu \mathrm{m}$ and $900 \mu \mathrm{m}$ thick 3D-tissues were successfully constructed. A clear brown line located in the central part of the tissues can be observed in the samples.

In the next step, we examined constructed sandwich samples using an immunostaining method. We observed the samples in several places and found that the vascular structures had spread well in the central part of the tissues (Figure 3(e)) 
in 10-1-10 samples (1 coat). As for the Other 10-1-10 and 20-1-20 samples constructed by collagen coated NHDFs ( 1 and 2 coats), because the thickness of these samples were too great to be observed using CLSM, we instead used a fluorescence imaging system (EVOS ${ }^{\circledR}$ FL Auto Cell Imaging System) to observe whole tissue on culture inserts. The fluorescence images are shown in Figure 3(c) and Figure 3(d), vascularized network structures were well spread in the tissues after 96 hours incubation. Compared to 3D tissues constructed using a mixing method, thicker network structures were observed, which may be because the distance between HUVECs in the sandwich samples were closer than in the mixing samples, leading to more chance for cell attachment for the vascular network structures formation. Both fluorescence images and histological results suggest that vascular network structures grow well at the desired location in the 3D-tissues using both the mixing method and the sandwich method.

\subsection{Oxygen partial pressure measurement}

From a previously published report, hypoxic conditions induce formation of endothelial vascular networks inside the tissues. To measure the hypoxic condition inside the constructed 3D-environment, the oxygen partial pressure in the media was measured during incubation (Figure S4). We evaluated oxygen partial pressure of 
10-1-10 3D-tissues and 3D-tissues without HUVECs (20 layers) inside and outside the culture inserts.

Oxygen partial pressure results shown in Figure S4(a) showing the results of 3D-tissues without addition of HUVECs revealed a slight reduction from $150 \mathrm{mmHg}$ to $120-130 \mathrm{mmHg}$ during 24 hours of incubation. The oxygen partial pressure remained stable even after changing the culture medium. As for the results for the 3D-tissues with HUVECs, the oxygen partial pressure decreased to $80-90 \mathrm{mmHg}$ during incubation, suggesting that addition of HUVECs inside the tissues dramatically decreased oxygen partial pressure. These results are consistent with the published results.

The values outside the culture inserts were also evaluated (Figure S4(b)). From the results, both tissues with and without addition of HUVECs remained stable (130-140 mmHg) even after 48 hours of incubation. This may be because the exchange of the culture medium inside and outside can only be accessed through the upper and lower parts of the culture inserts, making it difficult for it to exchange well during the incubation.

In this study, we successfully constructed 3D-tissues with specific cell types at a desired location by collagen coated cells. In this study, we applied two different construction methodologies: a mixing method and a sandwich culture method. In the 
case of the mixing method, with the increase in seeding cell numbers of HUVECs in the culture inserts, the increase of vascular network structures labelled by CD31 and fluorescent molecule were observed by fluorescence microscope. From the results of 3D-tissues constructed using the sandwich culture method, we observed vascular network structures at the central part using the fluorescence labelling and histological methods. From these two different construction systems, well spread or location-controlled network structures were both successfully constructed in approximately millimeter-sized tissues, indicating that the collagen coating method also provides the potential to construct 3D-tissue models similar to natural tissues, which may be useful in tissue engineering and pharmaceutical applications.

\section{Conflict of interest}

There is no conflict of interest.

\section{Acknowledgements}

This study was supported by the NEXT Program (LR026), a Grant-in-Aid for

Scientific Research (S) (232250040), a Grant-in-Aid for Scientific Research on 
Innovative Areas (26106717), a Grant-in-Aid for Scientific Research (B) (26282138) from MEXT of Japan, and the SENTAN-JST Program (13A1204). We would also like to thank Prof. Hiroshi Shimoda for his technical assistance of TEM observation, and Ms. Ayami Hiura for her technical assistance of histological HE staining. 


\section{References}

[1] M. Matsusaki, C.P. Case, M. Akashi, Three-dimensional cell culture technique and pathophysiology, Adv. Drug Deliv. Rev. 74 (2014) 95-103.

[2] N. Asakawa, T. Shimizu, Y. Tsuda, S. Sekiya, T. Sasagawa, M. Yamato, et al., Pre-vascularization of in vitro three-dimensional tissues created by cell sheet engineering, Biomaterials. 31 (2010) 3903-3909.

[3] L. Ren, D. Ma, B. Liu, J. Li, J. Chen, D. Yang, et al., Preparation of Three-Dimensional Vascularized MSC Cell Sheet Constructs for Tissue Regeneration, Biomed Res. Int. 2014 (2014) 1-10.

[4] K. Sakaguchi, T. Shimizu, T. Okano, Construction of three-dimensional vascularized cardiac tissue with cell sheet engineering, J. Control. Release. 205 (2015) 83-88.

[5] J.A. DeQuach, J.E. Lin, C. Cam, D. Hu, M.A. Salvatore, F. Sheikh, et al., Injectable skeletal muscle matrix hydrogel promotes neovascularization and muscle cell infiltration in a hindlimb ischemia model., Eur. Cell. Mater. 23 (2012) 400-412.

[6] P. G., T. M.V., C. M., Synthetic PEG hydrogel extracellular matrix mimics for the vascularization of engineered tissues, Cardiovasc. Pathol. 22 (2013) e31e32.

[7] J. Liu, H. Zheng, P. Poh, H.-G. Machens, A. Schilling, Hydrogels for Engineering of Perfusable Vascular Networks, Int. J. Mol. Sci. 16 (2015) 15997-16016.

[8] J.S. Miller, K.R. Stevens, M.T. Yang, B.M. Baker, D.-H.T. Nguyen, D.M. Cohen, et al., Rapid casting of patterned vascular networks for perfusable engineered three-dimensional tissues, Nat. Mater. 11 (2012) 768-774.

[9] Y. Zheng, J. Chen, M. Craven, N.W. Choi, S. Totorica, a. Diaz-Santana, et al., From the Cover: In vitro microvessels for the study of angiogenesis and thrombosis, Proc. Natl. Acad. Sci. 109 (2012) 9342-9347.

[10] H. Yoshida, M. Matsusaki, M. Akashi, Multilayered blood capillary analogs in biodegradable hydrogels for in vitro drug permeability assays, Adv. Funct. Mater. 23 (2013) 1736-1742.

[11] J. Tien, Microfluidic approaches for engineering vasculature, Curr. Opin. Chem. Eng. 3 (2014) 36-41.

[12] L.L.Y. Chiu, M. Radisic, Scaffolds with covalently immobilized VEGF and 
Angiopoietin-1 for vascularization of engineered tissues, Biomaterials. 31 (2010) 226-241.

[13] J.J. Moon, J.E. Saik, R.A. Poché, J.E. Leslie-Barbick, S.-H. Lee, A.A. Smith, et al., Biomimetic hydrogels with pro-angiogenic properties, Biomaterials. 31 (2010) 3840-3847.

[14] G. Decher, Fuzzy Nanoassemblies: Toward Layered Polymeric Multicomposites, Science (80-. ). 277 (1997) 1232-1237.

[15] G. Decher, J. Hong, J. Gutenberg-universitt, Buildup of Ultrathin Multilayer Films by a Self-assembly Process, 1 Consecutive Adsorption of Anionic and Cationic Bipolar Amphiphules on Charged Surfaces, Makromol. Chem., Macromol. Symp. 46 (1991) 321-327.

[16] M. Matsusaki, Development of Three-Dimensional Tissue Models Based on Hierarchical Cell Manipulation Using Nanofilms, Bull. Chem. Soc. Jpn. 85 (2012) 401-414.

[17] M. Matsusaki, S. Amemori, K. Kadowaki, M. Akashi, Quantitative 3D analysis of nitric oxide diffusion in a 3D artery model using sensor particles, Angew. Chem. Int. Ed. 50 (2011) 7557-7561.

[18] M. Matsusaki, K. Kadowaki, Y. Nakahara, M. Akashi, Fabrication of cellular multilayers with nanometer-sized extracellular matrix films, Angew. Chem. Int. Ed. 46 (2007) 4689-4692.

[19] A. Nishiguchi, H. Yoshida, M. Matsusaki, M. Akashi, Rapid Construction of Three-Dimensional Multilayered Tissues with Endothelial Tube Networks by the Cell-Accumulation Technique, Adv. Mater. 23 (2011) 3506-3510.

[20] A. Nishiguchi, M. Matsusaki, Y. Asano, H. Shimoda, M. Akashi, Effects of angiogenic factors and 3D-microenvironments on vascularization within sandwich cultures, Biomaterials. 35 (2014) 4739-4748.

[21] C. Chaubaroux, E. Vrana, C. Debry, P. Schaaf, B. Senger, J.C. Voegel, et al., Collagen-based fibrillar multilayer films cross-linked by a natural agent, Biomacromolecules. 13 (2012) 2128-2135.

[22] E. Leikina, M. V Mertts, N. Kuznetsova, S. Leikin, Type I collagen is thermally unstable at body temperature., Proc. Natl. Acad. Sci. U. S. A. 99 (2002) 1314-1318.

[23] J.R. Harris, A. Reiber, Influence of saline and $\mathrm{pH}$ on collagen type I fibrillogenesis in vitro: Fibril polymorphism and colloidal gold labelling, 
Micron. 38 (2007) 513-521.

[24] C.-Y. Liu, M. Matsusaki, M. Akashi, The construction of cell-density controlled three-dimensional tissues by coating micrometer-sized collagen fiber matrices on single cell surfaces, RSC Adv. 4 (2014) 46141-46144.

[25] J. Jokinen, E. Dadu, P. Nykvist, J. Käpylä, D.J. White, J. Ivaska, et al., Integrin-mediated cell adhesion to type I collagen fibrils, J. Biol. Chem. 279 (2004) 31956-31963.

[26] F.G. Giancotti, E. Ruoslahti, Integrin signaling., Science. 285 (1999) 10281032.

[27] C.-Y. Liu, M. Matsusaki, M. Akashi, Cell effects on the formation of collagen triple helix fibers inside collagen gels or on cell surfaces, Polym. J. 47 (2015) 391-399.

[28] C.-Y. Liu, M. Matsusaki, M. Akashi, Control of Cell-Cell Distance and Cell Densities in Millimeter-Sized 3D Tissues Constructed by Collagen Nanofiber Coating Techniques, ACS Biomater. Sci. Eng. 1 (2015) 639-645.

[29] E.C. Novosel, C. Kleinhans, P.J. Kluger, Vascularization is the key challenge in tissue engineering, Adv. Drug Deliv. Rev. 63 (2011) 300-311.

[30] T. Shimizu, H. Sekine, Y. Isoi, M. Yamato, A. Kikuchi, T. Okano, Long-term survival and growth of pulsatile myocardial tissue grafts engineered by the layering of cardiomyocyte sheets., Tissue Eng. 12 (2006) 499-507. 


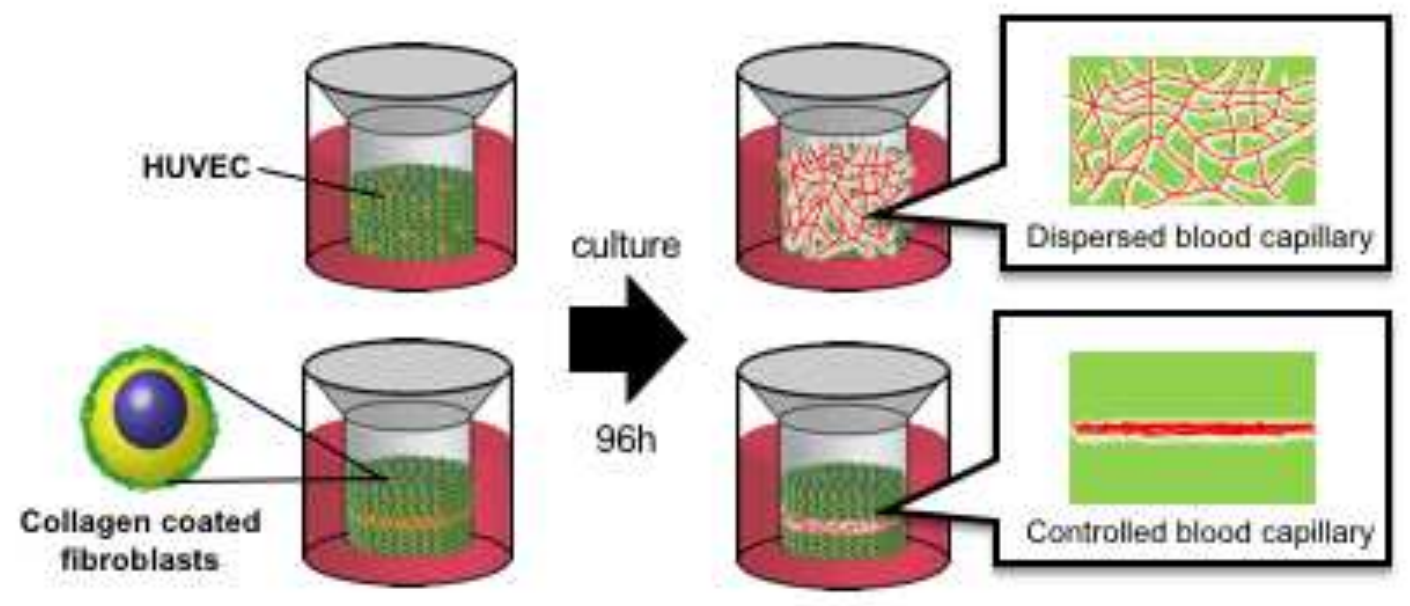

ollagen coat
fibroblasts 